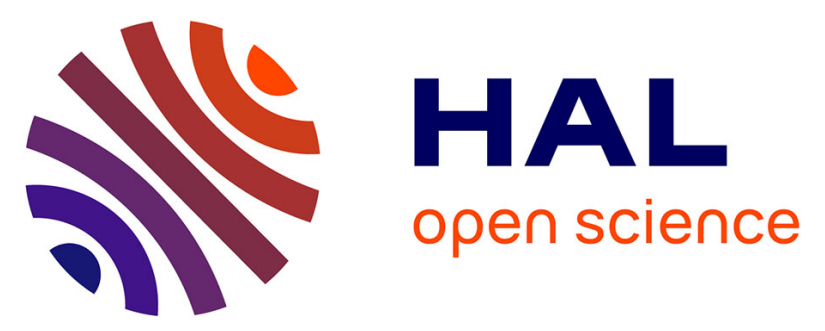

\title{
The modelling of the flow-induced vibrations of periodic flat and axial-symmetric structures with a wave-based method
}

\author{
F Errico, Mohamed Ichchou, S de Rosa, O. Bareille, F. Franco
}

\section{- To cite this version:}

F Errico, Mohamed Ichchou, S de Rosa, O. Bareille, F. Franco. The modelling of the flow-induced vibrations of periodic flat and axial-symmetric structures with a wave-based method. Journal of Sound and Vibration, 2018, 10.1016/j.jsv.2018.03.012 . hal-02090712

\section{HAL Id: hal-02090712 \\ https://hal.science/hal-02090712}

Submitted on 5 Apr 2019

HAL is a multi-disciplinary open access archive for the deposit and dissemination of scientific research documents, whether they are published or not. The documents may come from teaching and research institutions in France or abroad, or from public or private research centers.
L'archive ouverte pluridisciplinaire HAL, est destinée au dépôt et à la diffusion de documents scientifiques de niveau recherche, publiés ou non, émanant des établissements d'enseignement et de recherche français ou étrangers, des laboratoires publics ou privés. 


\title{
The modelling of the flow-induced vibrations of periodic flat and axial-symmetric structures with a wave-based method
}

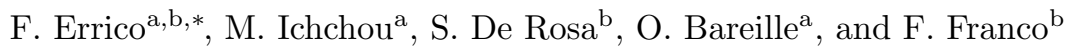 \\ ${ }^{a}$ LTDS, Laboratoire de Tribologie et Dynamique des Systems \\ Ecole Centrale de Lyon \\ ${ }^{b}$ pasta-lab, Laboratory for Promoting experiences in Aeronautical STructures and Acoustics \\ Dipartimento di Ingegneria Industriale - Sezione Aerospaziale \\ Universitá di Napoli Federico II
}

\begin{abstract}
The stochastic response of periodic flat and axial-symmetric structures, subjected to random and spatially-correlated loads, is here analysed through an approach based on the combination of a wave finite element and a transfer matrix method. Although giving a lower computational cost, the present approach keeps the same accuracy of classic finite element methods. When dealing with homogeneous structures, the accuracy is also extended to higher frequencies, without increasing the time of calculation. Depending on the complexity of the structure and the frequency range, the computational cost can be reduced more than two orders of magnitude. The presented methodology is validated both for simple and complex structural shapes, under deterministic and random loads. Keywords: Flow-induced vibrations, Periodic Structures, Wave Finite Element Method, Transfer Matrix Method, Turbulent Boundary Layer
\end{abstract}

\section{Introduction}

The vibrations, induced by a convective or an acoustic field exciting an elastic structure, arise strong interest in many engineering areas. These induced vibrations can even damage internal devices and payloads in the case of a space-launcher in the lift-off phase. The resulting radiated noise is, instead, a problem in the case of transport means in which the internal acoustic comfort can have a great importance: an aircraft fuselage, a train or vehicle cabin. As a result, the random vibrational and noise levels on the structures must be predicted in the design phase, in order to fix eventual issues before operating the system.

The response of fluid loaded structures can be estimated from data on wall pressure fields (WPF) and their correlation functions. The first characterisation of the wall pressure fluctuations has been carried out by Corcos [1, on experimental measurements. This model, which does not take into account the position of each point on the structure, considering just the relative distances, is one of the simplest and most used in literature. Specifically, the cross spectral density of the pressure fluctuations, through the assumption of separation of variables, is function of the relative distances among points, the convection velocity and the frequency.

* Corresponding author

Email address: fabrizio.errico@ec-lyon.fr (F. Errico) 
Many other models have been proposed to improve the accuracy of the Corcos model in the estimation of the WPFs (Wall Pressure Fluctuations) in the sub-convective domain, which is the wavenumber domain comprised between the acoustic and the convective wavenumber. Some of these models are directly linked to the Corcos one [2, 3], on the contrary, others do not take into account the separation of the stream-wise and cross-wise coherence [4, 5].

Most of the literature, regarding flow-induced vibrations, is limited to very simple cases, such as a flat rectangular simply-supported panel subjected to a specific type of fluid turbulence model. Even if this is a useful test-case, it is not industrially relevant and only few more complex applications are available. The predictive methodologies actually available in literature are mainly modal-based and energy-based [6, 7, 8, 9, 10].

Among the modal approaches, there is the full finite element approach, which makes use of a discretisation of the structural operator in finite elements, whose size is, generally, strongly affected by the fluid loading wavelength at the design frequency of analysis, [10]. This is, for most of applications, lower than the structural one, increasing, thus, the size of the problem in terms of degrees of freedom (DoF). Some authors proposed different methods to reduce the cost issues associated to this approach. For example, a method of load approximation through equivalent deterministic forces, analysing the eigenvalues distributions of the load matrix for a general convective load distribution, has been proposed in [11. Two frequency ranges are identified in which the convective load can be approximated through a purely coherent or purely incoherent equivalent load, proposing thus, a pseudo equivalent deterministic excitation method as an upgrade of the classic pseudo excitation method [1]. This approach proved to work very well in the low or high frequencies since the behaviour of the eigenvalues of the load matrix is easily predictable. In the mid frequencies, some approximations have to be made and the accuracy lowers [12].

Another possibility is to use scaled models or similitudes to reproduce the response of smaller systems, which have a reduced computational cost for given wavelength to describe, assuming fixed mesh sizes. In these cases, the main issues, are associated to the application of scaling laws to rebuild the response of the original system [8, 12.

On the other hand, moving to high-frequencies, energy-based approaches can be used. Among the methods proposed in literature, the most effective is surely the equivalent rain-on-the-roof method, [7]. This approach considers the fact that even spatially-correlated fluctuations, when moving to high-frequencies, head towards uncorrelated models. Then, it is possible to evaluate an equivalent uncorrelated load which approximates the real load correlation function. This gives huge advantages to the calculation of the joint acceptance integral, [7.

Most of these techniques have limits associated to the accuracy and the computational cost in certain frequency ranges, especially in the medium one.

This paper deals with the modelling of the flow-induced vibrations of periodic and axialsymmetric structures when random excitations act on the external surface of the structure by means of the pressure fluctuations, inducing vibrations and radiated noise inside the structural cavities. A wave-based methodology, within a transfer matrix framework, is proposed, in order 


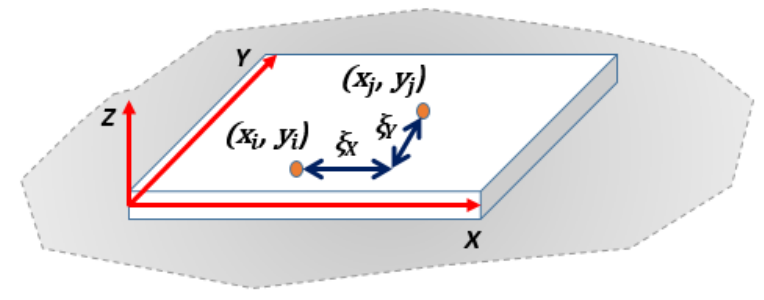

Figure 1: Sketch of the spatial correlation among two point in a structure

to reduce the computational cost. On the other hand, for a given computational cost, the frequency range of accuracy is extended overcoming the actual state of the art, in particular for axial-symmetric structures.

The work is structured as follows: Section 2 presents a theoretical background; Section 3 proposes and explains the methodologies and the new formulation for axial-symmetric structures. The final section presents the results for all the test cases.

\section{Theoretical background}

\subsection{The Fluid: Corcos Model}

The coherence model for the wall pressure fluctuations proposed by Corcos can be expressed through the product of two separate functions in the stream-wise and cross-wise directions, respectively:

$$
X_{p p}(\xi, \omega)=S_{p}(\omega) \Gamma\left(\xi_{x}, \xi_{y}, \omega\right)
$$

where

$$
\Gamma\left(\xi_{x}, \xi_{y}, \omega\right)=\mathrm{e}^{-\alpha_{x}\left|\omega \xi_{x} / U_{c}\right|} \mathrm{e}^{-\alpha_{y}\left|\omega \xi_{y} / U_{c}\right|} \mathrm{e}^{\mathrm{i}\left|\omega \xi_{x} / U_{c}\right|}
$$

$U_{c}$ is the convective flow speed, $S_{p}$ is the single-point auto spectral density of the wall pressure distribution, $\alpha_{x}$ and $\alpha_{y}$ are the correlation coefficients and $\xi_{x}$ and $\xi_{y}$ are the relative distances for the stream-wise and cross-wise directions respectively (Fig. 11. Some assumptions are present in this model as the separation of variables, the exponential form of the functions, the dependency on the distances instead of the point locations and the stream-wise harmonic variation accounted. It has to be underlined, also, that the correlation coefficients, $\alpha_{x}$ and $\alpha_{y}$, are to be determined from wind tunnel gallery measurements of the spatial coherence of the wall pressure fluctuations, which is, of course, a limit to its applicability.

However, this model is extensively used in literature as a predictive model and it is used in this paper too. This is due to the possibility to use a range of universal values for the correlation coefficients if the assumptions of no-gradients effects and fully developed turbulent boundary layer (TBL) are assumed. In any case, more than other models, the Corcos one is the best choice for simplicity versus predictive capabilities. 


\subsection{Diffuse Acoustic Field (DAF)}

This load model, which represents the excitation of an infinite sum of uncorrelated plane waves, has the following correlation function, depending on the convective constant only:

$$
\Gamma\left(\xi_{x}, \xi_{y}, \omega\right)=\frac{\sin \left(\omega \xi_{x} / U_{c}\right)}{\omega \xi_{x} / U_{c}} \frac{\sin \left(\omega \xi_{y} / U_{c}\right)}{\omega \xi_{y} / U_{c}}
$$

The DAF is often used to describe the TBL load in the low frequencies since both models take into account the stream-wise and cross-wise correlations. Moving up with the frequency the DAF model strongly overestimates the exciting contribution of a TBL.

In this paper, it is used to deal with cases in which the applicability of the Corcos model is impeded by the presence of pressure gradient effects 8 .

\subsection{Modal approaches for stochastic vibration analyses}

For a discretized approach, as standard FEM, the evaluation of the structural displacement cross-spectrum matrix to a statistically stationary and ergodic random excitation, such as TBL flow, can be performed as follows [6, 10, 12]:

$$
\mathbf{S}_{w w}(\omega)=\mathbf{\Psi} \mathbf{H}(\omega) \mathbf{\Psi}^{T} \mathbf{S}_{F F}(\omega) \mathbf{\Psi} \mathbf{H}^{*}(\omega) \mathbf{\Psi}^{T}
$$

where $\mathbf{H}$ being a complex diagonal matrix with:

$$
H_{j}(\omega)=\frac{1}{\omega_{j}^{2}-\omega^{2}+i \eta \omega_{j}^{2}}
$$

and $\boldsymbol{\Psi}$ is the real matrix of the modal shapes, $\mathbf{S}_{F F}$ is the load matrix in discrete coordinates. The superscripts $T$ and $*$ denote, respectively, the matrix transposition and the conjugation. The output matrix $\mathbf{S}_{w w}$ is the matrix of the auto and cross spectral densities of the structural field.

Each element of the load matrix has to be calculated solving a double integral on the nodal surface of the product of the structural shape functions $\left(N_{F}\right)$ and the wall pressure cross spectra as in Eq. (6):

$$
S_{F F}^{i, j}=\int_{x_{i}-\Delta_{x} / 2}^{x_{i}+\Delta_{x} / 2} \int_{y_{i}-\Delta_{y} / 2}^{y_{i}+\Delta_{y} / 2} \int_{x_{j}-\Delta_{x} / 2}^{x_{j}+\Delta_{x} / 2} \int_{y_{j}-\Delta_{y} / 2}^{y_{j}+\Delta_{y} / 2} N_{F}^{T} X_{p p}\left(\xi_{x}^{i j}, \xi_{y}^{i j}, \omega\right) N_{F} d x_{i} d x_{j} d y_{i} d y_{j}
$$

The size of the finite elements is, generally, strongly ruled by the fluid wavelength at the design frequency of analysis. For most of applications, this is lower than the structural one and the need of a finer mesh increases the size of the problem and, thus, the computational cost. For example, with reference to Fig,2, a structural mesh sized on $10 \mathrm{KHz}$ is able to describe the fluid wavelength only up to $2 \mathrm{KHz}$. It is clear how, moving up with the frequency, the computational cost increases more and more. In Fig 2 it is also identifiable the critical frequency which is the point where the fluid wavelength equals the structural one. This double space integration on the wetted (excited) surface (Eq. (6)), can be approximated trying to reduce the time associated to this analysis step. Considering that the pressure fluctuations, due to TBL, do not fluctuate very quickly in the nodal area, given by $\Delta_{x} \Delta_{y}$, a direct lump-on-the-nodes of the wall pressure cross spectra is a good approximation of the integral, as in Eq.(7) [9, 10, 12]:

$$
S_{F F_{L}}^{i, j}=X_{p p}\left(\xi_{x}^{i j}, \xi_{y}^{i j}, \omega\right) \Delta_{x}^{2} \Delta_{y}^{2}
$$




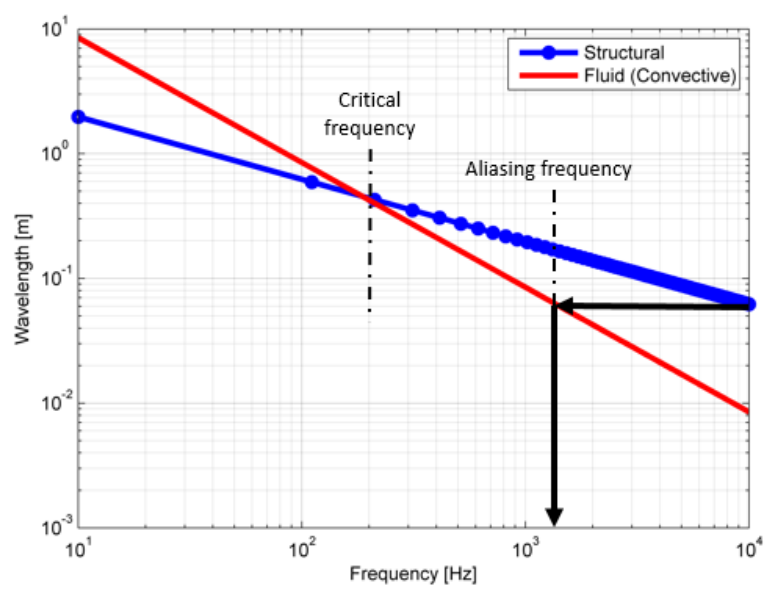

Figure 2: The structural and aerodynamic wavelengths versus frequency - Flow speed $U_{0}=100 \mathrm{~m} / \mathrm{s}$

This approximation is always true if the frequency range is low or the nodal area is small enough to guarantee that the pressure is effectively not fluctuating much in the considered spatial domain. Thus, once a mesh is defined, it is straightforward to evaluate the maximum frequency where the fluid pressure fluctuations are well described, which is called aliasing frequency. Overcoming this value, the load matrix is no more an accurate discretization of the pressure fluctuations and the model exponentially loses accuracy. A deep investigation on this numerical aspects is extensively made in literature, [10].

This numerical finite element-based approach is used as a reference for the results of this paper, when an analytic approach in not available. These are, in fact, available only for flat simply-supported panels subjected to Corcos load 9 .

\section{The Proposed Method}

The following approach is based on a combination of the transfer matrix method with a wave finite element method for periodic structures. A new formulation is proposed in order to deal with cyclic periodic structures.

\subsection{The Transfer Matrix Method}

The method uses of a transfer matrix between the fluid excitation and specific target degrees of freedom. This is built through the evaluation of the Green functions of each target DoF. Assuming a field of interest $v$ (displacement, velocity, acceleration, pressure etc.) the response to the random load can be calculated as such:

$$
S_{v v}\left(x_{i}, x_{j}, \omega\right)=\boldsymbol{\Theta}_{\boldsymbol{v}}^{T}\left(x_{i}, \omega\right) \mathbf{S}_{F F}(\omega) \boldsymbol{\Theta}_{\boldsymbol{v}}\left(x_{j}, \omega\right)
$$

Where $\boldsymbol{\Theta}_{v}$ is the transfer matrix, which is a $m \mathrm{x} t$ matrix, and $S_{v v}$ is the matrix of the auto/cross spectral densities of the field $v$. The value of $t$ is associated to the target DoFs while the value of $m$ is dependent on the fluid mesh (wetted surface). This method gives an immediate advantage in 
the possibility to easily choose the value of $t$ depending on the analysis needs; it can be even one or the total number of DoFs of the model.

It is important to point out that the values of $t$ and $m$, apart from being flexible and objectivesoriented, are always inferior to the total number of degrees of freedom of the whole FE model. Moreover, the non-wetted degrees of freedom do not increase the problem size, even if included in the system dynamics when computing the transfer matrix, being able to choose the values of $t$ and $m$ almost arbitrary.

The elements $\Theta_{v}^{i, j}$ of the transfer matrix are values of the transfer Green function between the two points $i$ and $j$. This paper proposes to numerically calculate these elements through the a wave-based technique and the Maxwell-Betti reciprocal work theorem; This theorem states that for a linear elastic structure subjected to two sets of forces $P$ and $Q$ the work done by the set $P$ through the displacements produced by the set $Q$ is equal to the work done by the set $Q$ through the displacements produced by the set $P$.

\subsection{The Wave Finite Element Method (WFEM)}

The adopted wave-based technique is a wave finite element (WFE) method applicable for homogeneous and periodic structures. The method makes use of the periodic conditions to derive, through an eigenvalue problem, the wavemodes of the periodic media. The solution is based on a wavemode approach, in which the problem is analysed through the superposition of different wavefields, [13, 14, 15, 16]. The method perfectly fits our need to calculate the Green functions due to its flexibility.

Considering a single repetitive substructure of our periodic media, the waves and their crosssectional waveshapes (wavemodes), travelling in the structure, can be calculates by solving the eigenproblem which derives from imposing the Floquet's conditions (periodicity). In the 1D case the periodicity conditions are defined by imposing the equilibrium of the displacement and forces among the common hypernodes of two subsequent periodic cells (Fig. 33).

Starting from the dynamic stiffness matrix problem of a partial assemble of cells, reordering the DoFs so that internal, left and right nodes's degrees of freedom are properly separated, one can move to the eigenvalue problem imposing the periodic conditions, assuming time and space harmonic excitation. Damping can be modelled by viscous damping matrix $\mathbf{C}$ or by $\mathbf{K}$ being complex. In the following equations, the left and right side hypernodes are defined with the letters $L$ and $R$ respectively:

$$
\left[\begin{array}{lll}
\mathbf{D}_{l l} & \mathbf{D}_{l r} & \mathbf{D}_{l i} \\
\mathbf{D}_{r l} & \mathbf{D}_{r r} & \mathbf{D}_{r i} \\
\mathbf{D}_{i l} & \mathbf{D}_{i r} & \mathbf{D}_{i i}
\end{array}\right]\left\{\begin{array}{l}
\mathbf{q}_{L} \\
\mathbf{q}_{R} \\
\mathbf{q}_{I}
\end{array}\right\}=\left\{\begin{array}{c}
\mathbf{F}_{L} \\
\mathbf{F}_{R} \\
\mathbf{F}_{I}
\end{array}\right\}
$$

where $\mathbf{D}=\left[\mathbf{K}-\omega^{2} \mathbf{M}\right]$. Condensing the internal degrees of freedom (I), substituting the third equation of Eq. (9), in the first two, one obtains his base-work equation:

$$
\left[\begin{array}{ll}
\mathbf{D}_{L L} & \mathbf{D}_{L R} \\
\mathbf{D}_{R L} & \mathbf{D}_{R R}
\end{array}\right]\left\{\begin{array}{l}
\mathbf{q}_{L} \\
\mathbf{q}_{R}
\end{array}\right\}=\left\{\begin{array}{l}
\mathbf{F}_{L} \\
\mathbf{F}_{R}
\end{array}\right\},
$$




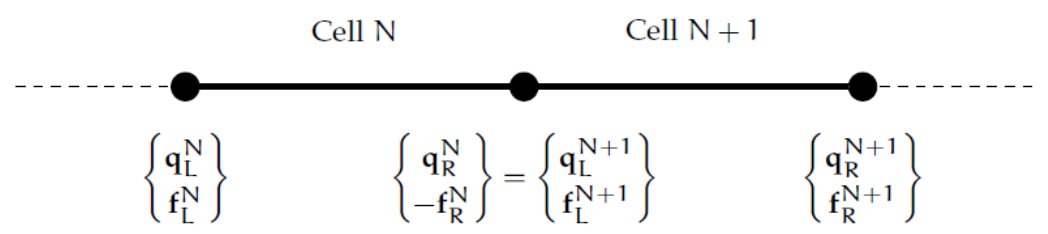

Figure 3: Two adjacent elementary cells of a waveguide 22]

with:

$$
\begin{array}{r}
\mathbf{D}_{B B}=\mathbf{D}_{B B}-\mathbf{D}_{B i} \mathbf{D}_{i i}^{-1} \mathbf{D}_{i B} \\
\mathbf{F}_{B}=\mathbf{F}_{B}-\mathbf{D}_{B i} \mathbf{D}_{i i}^{-1} \mathbf{F}_{I}
\end{array}
$$

where $B$ stands for cell boundary, left or right. Eq. (11) is general, but in the cases here analysed, the forces on the inner nodes are considered null. This is valid within the framework of homogenized structures and it is used in this paper for validation purposes. Other techniques can be used to condense the inner nodes, as a Craig-Bampton (CB) dynamic condensation method. This is strongly suggested for complex cross-sectional shapes [17, 18, 19].

Imposing continuity of displacements and equilibrium of forces at the interface between adjacent points and putting all in a matrix, the Transfer matrix, $\mathbf{T}$, can be derived, $15,16,20,21$. It relates the nodal displacements and forces (evaluated on the left side) between two adjacent substructures:

$$
[\mathbf{T}]=\left[\begin{array}{cc}
-\mathbf{D}_{L R}^{-1} \mathbf{D}_{L L} & \mathbf{D}_{L R}^{-1} \\
-\mathbf{D}_{R L}+\mathbf{D}_{R R} \mathbf{D}_{L R}^{-1} \mathbf{D}_{L L} & -\mathbf{D}_{R R} \mathbf{D}_{L R}^{-1}
\end{array}\right] .
$$

It has been demonstrated that the eigenvalues of the transfer matrix occur in reciprocal pairs as $\lambda_{j}^{+}=1 / \lambda_{j}^{-}$corresponding to pairs of positive (+) and negative (-) going waves, respectively, [13, 14, 15]. The positive and negative going eigenvectors $\phi_{j}^{-}$and $\phi_{j}^{+}$are associated to the above eigenvalues. These eigenvectors are referred to as wavemodes, which are the displacement and force distributions in the substructure section [18, 21, 23. Every wavemode can be partitioned into a sub-vector of DoFs, $\boldsymbol{\Phi}_{q}^{+,-}$, and internal forces/moments, $\boldsymbol{\Phi}_{f}^{+,-}$.

Positive waves are characterized by the magnitude of the propagating constant being inferior to unity $\left(\left|\lambda_{j}^{+}\right|<1\right)$, which means that if the wave propagates its amplitude must decrease in travelling. If $\left|\lambda_{j}^{+}\right|=1$ then the time average power transmission in the positive direction is evaluated to select the positive and negative going waves.

A transformation between the physical domain, where the system behaviour is described in terms of $\mathbf{q}$ and $\mathbf{f}$, and the wave domain, where the behaviour is described in terms of waves of amplitudes $\mathbf{a}^{+}$ans $\mathbf{a}^{-}$travelling in the positive and negative directions, is derived through these matrices [17, 19, 24]. In particular

$$
\left\{\begin{array}{c}
\mathbf{q}_{L} \\
\mathbf{f}_{L}
\end{array}\right\}=\left[\begin{array}{ll}
\boldsymbol{\Phi}_{q}^{+} & \boldsymbol{\Phi}_{q}^{-} \\
\boldsymbol{\Phi}_{f}^{+} & \boldsymbol{\Phi}_{f}^{-}
\end{array}\right]\left\{\begin{array}{l}
\mathbf{a}^{+} \\
\mathbf{a}^{-}
\end{array}\right\}
$$

where $\mathbf{a}^{+,-}$are the wave amplitudes of the positive and negative going waves. It is important to emphasize that the wavemode-basis needs a filtering process to avoid numerical issues in the 


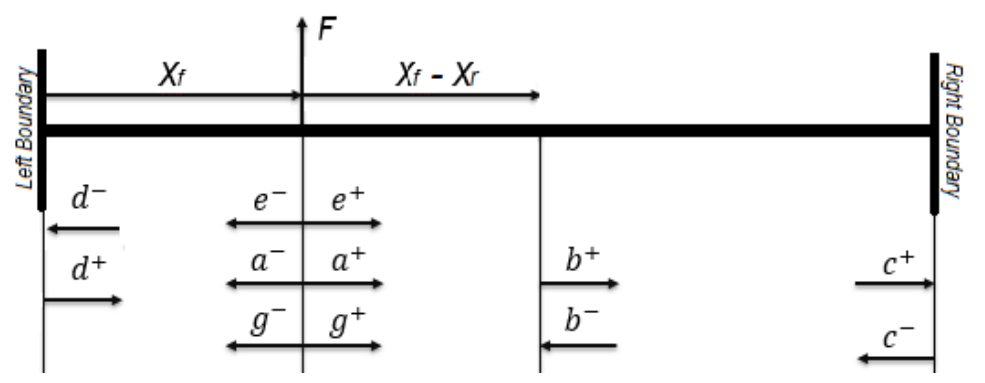

Figure 4: Waves in a finite structure excited by point load

analysis. This is mainly addressable to the presence of waves which do not propagate in a certain frequency range but which are still present in the base as complex or purely imaginary wavenumbers. Mantaining these components in the wavebase is not a conceptual mistake but it generally gives numerical conditioning. Physically, this means considering the only contribution, to structural response, of the propagating or close-to propagating waves. At each frequency step, when and if new wavemodes cut-on, these are included in the wavemode-basis.

With reference to Fig. 4, the forced vibrations in a response point can be evaluated through the superimposition of the direct and reverberant field. The first is the result of the excitation and the second is the incident wavefield.

The continuity of displacements and the equilibrium of the forces at the excitation point using the wave base expansion, can be expressed in matrix form:

$$
\left[\begin{array}{cc}
\boldsymbol{\Phi}_{q}^{+} & -\boldsymbol{\Phi}_{q}^{-} \\
\boldsymbol{\Phi}_{f}^{+} & -\boldsymbol{\Phi}_{f}^{-}
\end{array}\right]\left\{\begin{array}{l}
\mathbf{e}^{+} \\
\mathbf{e}^{-}
\end{array}\right\}=\left\{\begin{array}{r}
\mathbf{0} \\
\mathbf{f}_{e x t}
\end{array}\right\},
$$

where $\mathbf{e}^{+,-}$is the vector of the amplitudes of the wavemodes in the direct field. The inversion of the above left-hand side matrix can lead to numerical errors, especially for complex structures and cell shapes. A solution consists in exploiting the orthogonality of the left and right eigenvectors; i.e. pre-multiplying both sides of the previous equation by the matrix of left eigenvectors properly rearranged to get to the following form:

$$
\left\{\begin{array}{l}
\mathbf{e}^{+} \\
\mathbf{e}^{-}
\end{array}\right\}=\left\{\begin{array}{rr}
\boldsymbol{\Psi}_{f}^{+} & \mathbf{f}_{\text {ext }} \\
-\mathbf{\Psi}_{f}^{-} & \mathbf{f}_{\text {ext }}
\end{array}\right\} .
$$

Of course, this requires a left-eigenvalue problem, whose eigenvectors are $\Psi^{+,-}$, to be solved too. It is worth to underline how the use of the force wavemodes is different from the use of the displacement ones, shown by Waki et al.[16], and it has proved to lead to accurate results.

To take into account the reverberant field, the reflection, transmission and absorption of waves incident upon discontinuities and boundaries must be investigated, as their propagation. In this paper, the sole reflection and propagation are taken into account.

Considering a generic wave of amplitude $\mathbf{h}^{+}$travelling in the medium, one can model the reflection and the subsequent opposite-going wave amplitude, $\mathbf{h}^{-}$, with the use of reflection matrices at boundaries. Given $\mathbf{R}$ as the matrix of reflection coefficients, which depends on the type of 
constraint, the wave problem at the boundaries can be expressed as $\mathbf{h}^{+,-}=\mathbf{R}_{l, r} \mathbf{h}^{-,+}$. Each boundary condition can always be expressed in the form: $\mathbf{A f}+\mathbf{B q}=\mathbf{0}$. Substituting and using the wave-base expansion:

$$
\begin{gathered}
\mathbf{R}_{r}=-\left(\mathbf{A} \Phi_{f}^{-}+\mathbf{B} \Phi_{q}^{-}\right)^{-1}\left(\mathbf{A} \boldsymbol{\Phi}_{f}^{+}+\mathbf{B} \Phi_{q}^{+}\right) \\
\mathbf{R}_{l}=-\left(\mathbf{A} \Phi_{f}^{+}+\mathbf{B} \boldsymbol{\Phi}_{q}^{+}\right)^{-1}\left(\mathbf{A} \Phi_{f}^{-}+\mathbf{B} \Phi_{q}^{-}\right)
\end{gathered}
$$

where the matrices $\mathbf{A}$ and $\mathbf{B}$ are dependent on the type of constrain. In the case of force-free boundaries, for example, $\mathbf{A}=\mathbf{I}$ and $\mathbf{B}=\mathbf{0}$.

At the same time, moving in the medium, the amplitude of all the waves changes, depending on the distance and the wave characteristics itself. Using the periodicity features the waves amplitudes at a distance $x$ from a given reference can be expressed through the wave propagation matrix, $\operatorname{Tr}(x)$.

$$
\operatorname{Tr}(x)=\operatorname{diag}\left(e^{-\mathrm{i} k_{1} x}, e^{-\mathrm{i} k_{2} x}, \ldots, e^{-\mathrm{i} k_{n} x}\right) .
$$

All the elements of the wave propagation matrix have a magnitude less or equal to the unity, by definition.

Once the amplitudes of the direct excited waves are known, the waves amplitudes, at a given response point, can be calculated by considering the excitation, reflection and propagation relations obtained so far.

Using again Fig. 4 as reference, the amplitude of waves in the reference point can be evaluated; given $L$ the length of the whole waveguide, $x_{f}$ the coordinate of the excitation point:

$$
\begin{array}{r}
\mathbf{a}^{+}=\left[\mathbf{I}-\operatorname{Tr}\left(x_{f}\right) \mathbf{R}_{l} \operatorname{Tr}(L) \mathbf{R}_{r} \operatorname{Tr}\left(L-x_{f}\right)\right]^{-1}\left[\mathbf{e}^{+}+\operatorname{Tr}\left(x_{f}\right) \mathbf{R}_{l} \operatorname{Tr}\left(x_{f}\right) \mathbf{e}^{-}\right] \\
\mathbf{a}^{-}=\left[\mathbf{I}-\operatorname{Tr}\left(L-x_{f}\right) \mathbf{R}_{r} \operatorname{Tr}(L) \mathbf{R}_{l} \operatorname{Tr}\left(x_{f}\right)\right]^{-1}\left[\mathbf{e}^{-}+\operatorname{Tr}\left(L-x_{f}\right) \mathbf{R}_{r} \operatorname{Tr}\left(L-x_{f}\right) \mathbf{e}^{+}\right]-\mathbf{e}^{-}
\end{array}
$$

The adopted approach guarantees the numerical stability. In fact, the above solutions are wellconditioned because the matrices, to be inverted, are diagonally dominant and the elements of the wave propagation matrices are less than or equal to the unity. The response in the reception point can be then calculated applying the propagation relations 24].

\subsection{An axial-symmetric WFE formulation (AsWFE)}

A WFE formulation is here presented in order to deal with complex axial-symmetric structures. The main issue of the standard WFE approach relies in the complexity in dealing with tapered structures since each subsection is different from the previous one. This is the case of cones, where, moving along the axis, each point behaves on a circumference of different radius, or complex shapes as space launcher fairings.

The first idea is to simulate the curvature of our substructure through the rotation of the degrees of freedom at the borders, as shown in Fig. 5] 25].

A rotational matrix $\mathbf{r}$ is defined and assembled in a block diagonal matrix, Rot. It is intended 


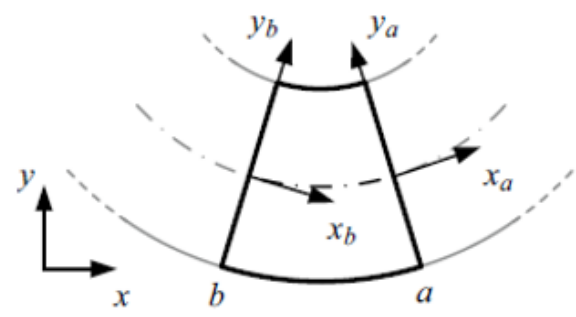

Figure 5: The rotation of the global reference to the substructure borders [25]

to be done for each curvature; in this case a single curvature is considered.

$$
\boldsymbol{R o t}_{j}=\left[\begin{array}{cccc}
\mathbf{r}_{j} & 0 & \ldots & 0 \\
0 & \mathbf{r}_{j} & \ldots & 0 \\
\vdots & & & \vdots \\
0 & \ldots & & \mathbf{r}_{j}
\end{array}\right]
$$

Hence, the mass and stiffness matrices of the curved waveguide are obtained as:

$$
\begin{gathered}
\mathbf{M}=\boldsymbol{\operatorname { R o t }}_{j}^{T} \mathbf{M}_{\text {flat }} \boldsymbol{R o t}_{j} \\
\mathbf{K}=\boldsymbol{\operatorname { R o t }}_{j}^{T} \mathbf{K}_{\text {flat }} \boldsymbol{R o t}_{j} .
\end{gathered}
$$

Imposing the periodicity conditions, to move to the eigenvalue problem to calculate the wavemodes of the waveguide, as in Eq. $[12$, the wave propagation is automatically considered along the curved path. The results are the circumferential wavemodes.

Moreover, considering the known relationships among arcs and angles, it is easy to analyse the wave propagation using the circumferential wavenumber and the angular distance between points as parameters. This is useful since, for tapered structures, different points run different lengths along the curved path, causing some technical issues. The presented formulation is more general and for each circumferential wave one can write:

$$
\lambda_{\theta}=e^{-\mathrm{i} k_{\theta} \Delta \theta}
$$

where $k_{\theta}=k_{x} R=k_{x} \Delta_{x} / \Delta \theta$ is the circumferential wavenumber and $\Delta \theta$ is the angular distance. As previously done for flat waveguides, the response in a target point can be calculated considering the superimposition of an equivalent direct field and the reverberant one, taking into account that, in the case of closed axial-symmetric structures, no real boundaries are present. For example, with reference to the scheme in Fig 6 , assuming a case in which $\Delta \theta>0$, the reverberant field is simply given by:

$$
\mathbf{g}^{+}=\operatorname{Tr}(2 \pi) \mathbf{b}^{+} \quad \mathbf{g}^{-}=\operatorname{Tr}(2 \pi-\Delta \theta) \mathbf{b}^{-}
$$

\subsection{Variable mesh}

The use of the WFE approach gives many advantages in building the transfer matrix $\boldsymbol{\Theta}_{v}$. In particular, in Eq. 17 , it is clear how the wave propagation can be taken into account using 


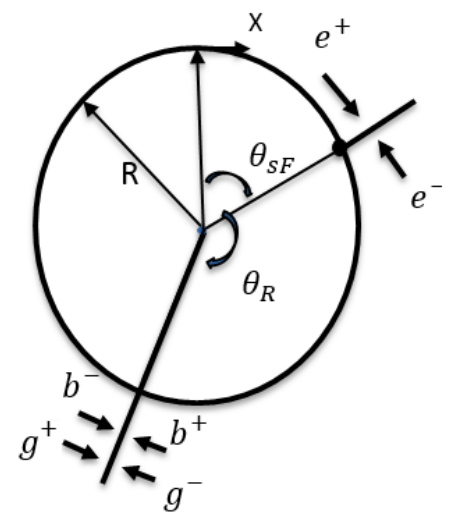

Figure 6: Waves in an axial-symmetric structure excited by a point load

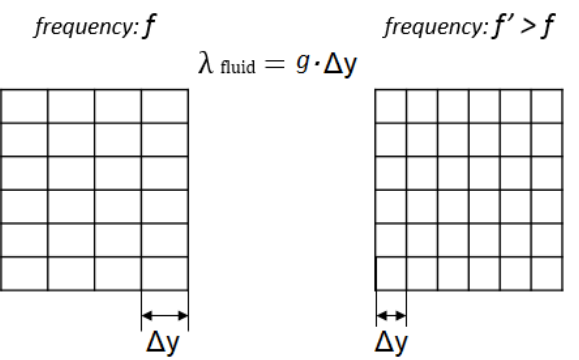

Figure 7: Mesh adaptation scheme

an analytic system of coordinates. This opens to the possibility of using variable meshes in the periodicity direction, evaluating the response in more intermediate point which do not strictly belong to the starting FE mesh. The value of $m$, connected to the mesh size on the wetted (external) surface, can be changed frequency-by-frequency imposing a different spatial step $(\Delta y)$, allowing a strong save in computational cost, with respect to the cases in which the mesh is initially set on the finest value. The size of the transfer matrix is, then, tuned on the fluid wavelength variation (i.e. versus the frequency of interest). In Fig 7, a simple scheme illustrating this operation, with $g$ as an integer generally comprised between 6 and 10 to correctly describe the desired wavelength.

A single coarse substructure can be used to analyse the dynamic problem to higher frequencies, instead of creating a finer representation of it through FE codes. The immediate results of this approach are evident when dealing with the stochastic response to random load. First of all the aliasing frequency moves forward enlarging the frequency range of accuracy for the approximation in Eq.(7). Then, given a frequency range of analysis, the computational advantage is higher and higher approaching the lower frequency limit of the band, since an equivalent coarser grid can be considered. In next section, the effects of this numerical property is shown with a specific test-case. 


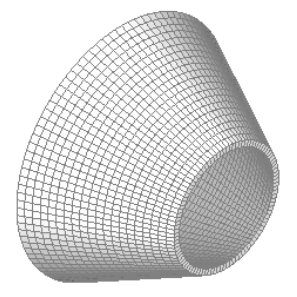

a)

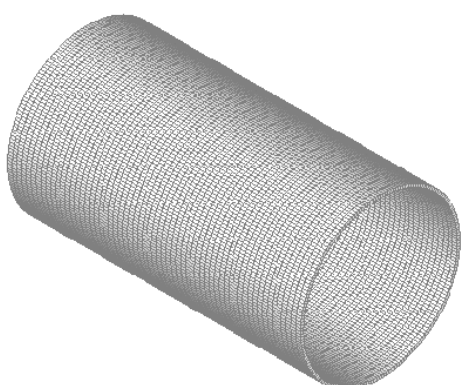

b)

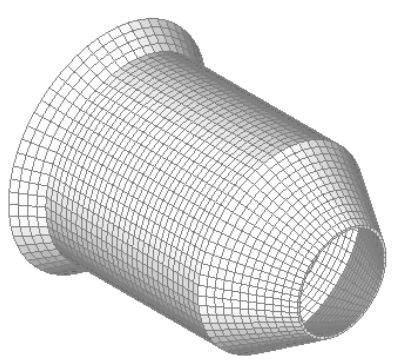

c)

Figure 8: Axial-symmetric Models analysed - (a) Conical; (b) Cylindrical; (c) Complex - SYLDA
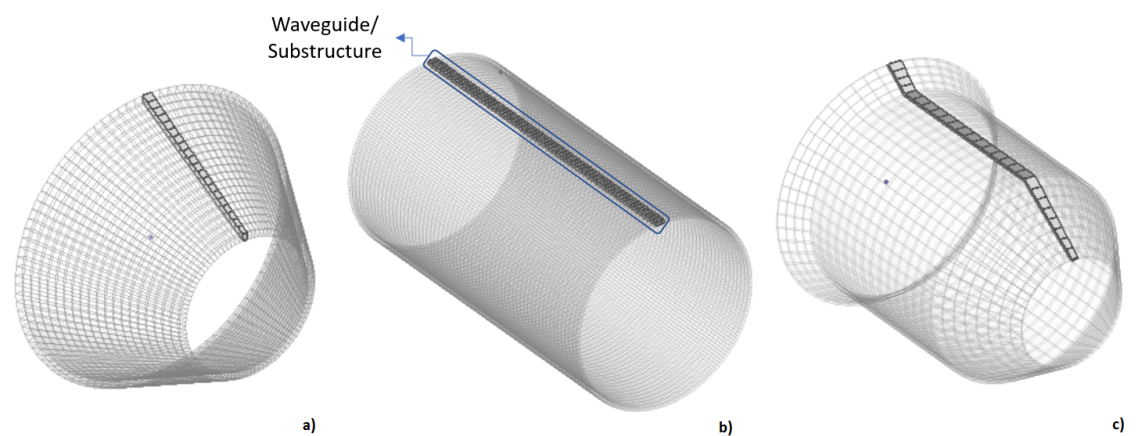

Figure 9: The relative substructures of the axial-symmetric models - (a) Conical; (b) Cylindrical; (c) Complex SYLDA

\section{Validating Results}

\subsection{Deterministic Response: Green Functions}

The above-described wave based methodologies are the instrument to calculate the Green functions of each target degree of freedom with respect to the wetted points, which are the nodes where the stochastic load is translated. In this section, a series of results for axial-symmetric structures are presented to assess the accuracy of the presented methodology. It is worth to remind that, using the transfer matrix method, the accuracy validation of the deterministic response leads also to the validation of the stochastic one.

The validation of the forced response to point load for flat structures, i.e. panels, using the standard WFE technique has been already done by many authors in literature [15, 16, 20, 21, 24. For this reason, here, a validation of the AsWFE (Axial-symmetric Wave Finite Element) method is illustrated.

Three basic axial-symmetric geometries are analysed: cylinder, cone and a complex assembly of them (Fig. 8). The latter is a scaled model of the SYLDA (SYstme de Lancement Double Ariane) structure, generally used in the Ariane V launcher for multiple simultaneous launch [23]. In any case, the structures are homogenized and made in aluminium. For sake of completeness, the geometrical properties of the models are reported in Table 1.

A full FE model is used in each case, as a reference, to compare and validate the numerical results. All the structures are modelled and the mass and stiffness matrices are extracted using a 
Table 1: Axial-symmetric models geometrical parameters

\begin{tabular}{cccc}
\hline Data & Conical & Cylindrical & Complex - SYLDA \\
\hline Length $[\mathrm{m}]$ & 0.20 & 0.6 & 0.40 \\
Diameter at $x=0[\mathrm{~m}]$ & 0.20 & 0.40 & 0.15 \\
Diameter at $x=\mathrm{L}[\mathrm{m}]$ & 0.40 & 0.40 & 0.34 \\
Diameter at $x=\mathrm{L} / 2[\mathrm{~m}]$ & 0.30 & 0.40 & 0.29 \\
Skin thickness $[\mathrm{mm}]$ & 4.0 & 4.0 & 7.0 \\
\hline
\end{tabular}

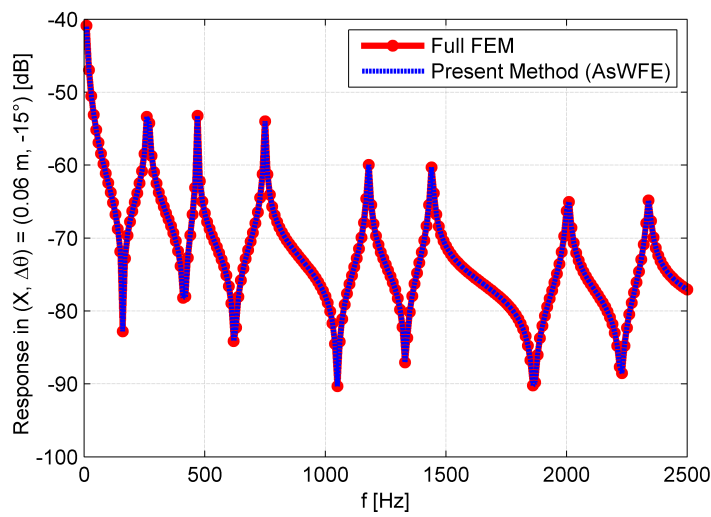

Figure 10: Mobility on the cylindrical model [dB ref. $1 \mathrm{~m} / \mathrm{s}$ ] - Numerical comparison between the present technique (AsWFE) and the full FEM for an impulsive nodal excitation.

commercial code (ANSYS). A force is applied in $(x, \Theta)=(L / 2,0)$, in all the cases, where $x$ and $\Theta$ are the axial and circumferential coordinates. The mobility response is computed in any point of the mesh grid. In Fig. 10, 11 and 12, the frequency response functions, in different specific points ( $\Delta_{x}$ and $\Delta_{\theta}$ the coordinates, with respect to the point of application of the force), are compared for the present method and the full FEM. Both circumferential and coupled modes, typical for axial-symmetric structures, are identified and precisely described (Fig. 13) and it is evident how the proposed methodology provides very accurate results with the highest relative error, inside the whole frequency band, inferior to $10^{-2} \mathrm{~dB}$.

Another advantage is also linked to the possibility of releasing the mesh constraints in the direction of the wave propagation, keeping the same accuracy of the FEM.

\subsection{Stochastic Response of Flat Structures: Panel Subjected to TBL}

A flat panel is considered, whose geometrical parameters are in Table 2, under a turbulent boundary layer load (Corcos model; $\alpha_{x}=0.15 ; \alpha_{y}=0.77 ; U_{c}=120 \mathrm{~m} / \mathrm{s}$ ). The panel is made in aluminium: $E=7.010^{10} \mathrm{~Pa}, \rho=2750 \mathrm{~kg} / \mathrm{m}^{3}, \nu=0.33$ and $1 \%$ of structural damping. The analytic solution is used as a reference and a comparison between a full FEM and the proposed method is shown. The analytic approach can be developed only for a flat rectangular panel simplysupported to the edges, as it is in this case. The final equation is here shown for more clearness 


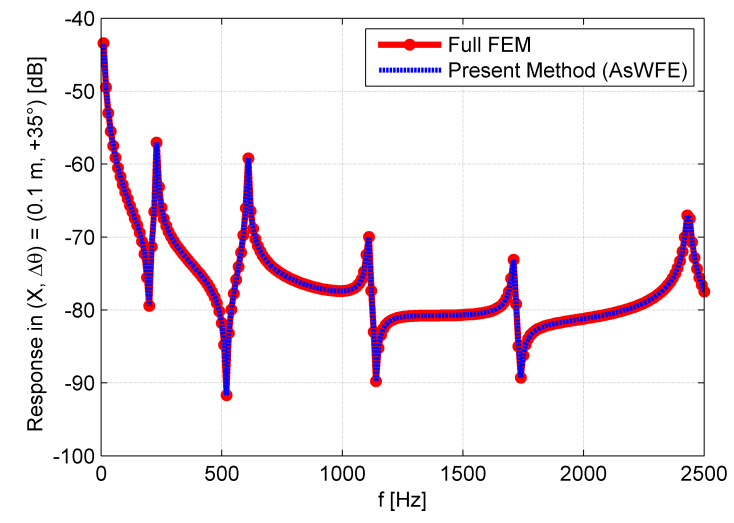

Figure 11: Mobility on the conical model [dB ref. $1 \mathrm{~m} / \mathrm{s}$ ] - Numerical comparison between the present technique (AsWFE) and the full FEM for an impulsive nodal excitation.

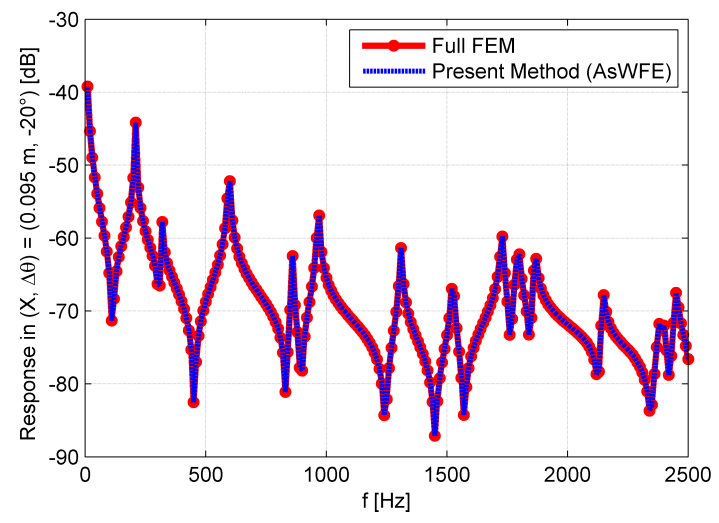

Figure 12: Mobility on the SYLDA model [dB ref. $1 \mathrm{~m} / \mathrm{s}$ ] - Numerical comparison between the present technique (AsWFE) and the full FEM for an impulsive nodal excitation.
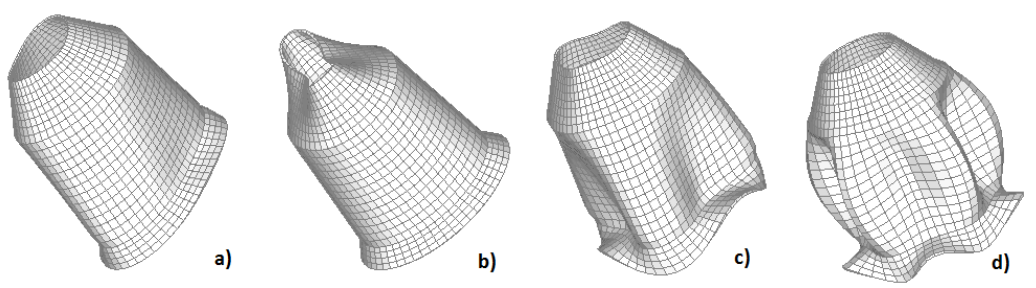

Figure 13: The circumferential and coupled modes for the SYLDA test-case; (a) $229.6 \mathrm{~Hz}$, (b) $357.2 \mathrm{~Hz}$, (c) 640.2 $\mathrm{Hz}$, (d) $984.5 \mathrm{~Hz}$ 
Table 2: Panel geometrical parameters

\section{Panel Geometrical Data}

$\begin{array}{cc}\text { Length stream-wise }(x)[\mathrm{m}] & 0.36 \\ \text { Length cross-wise }(y)[\mathrm{m}] & 0.20 \\ \text { Skin thickness }[\mathrm{mm}] & 3.0\end{array}$

Table 3: Computational cost comparison for a flat panel to TBL load

\begin{tabular}{|c|c|c|}
\hline Method & Design frequency & Elapsed time/frequency $[\mathrm{sec}]$ \\
\hline FEM - Eq. 44 and Eq. $\sqrt{7}$ & $6 \mathrm{kHz}$ & 82.65 \\
\hline WFE - Eq. 80 and Eq. 77 & $6 \mathrm{kHz}$ & 3.25 \\
\hline WFE - TM and variable mesh & $6 \mathrm{kHz}$ in $y$; variable in $x$ & 1 \\
\hline
\end{tabular}

[12:

$$
\mathbf{S}_{w w}\left(x_{a}, y_{a}, \omega\right)=\sum_{j} \sum_{n}\left[\frac{\Psi_{j}\left(x_{a}, y_{a}\right) \Psi_{n}\left(x_{a}, y_{a}\right)}{Z_{j}^{H}(\omega) Z_{n}(\omega) \gamma_{j} \gamma_{n}}\right] A_{Q_{j} Q_{n}}(\omega)
$$

where $\Psi_{j}$ is the jth analytic mode shape, $\gamma_{j}$ is the generalized mass coefficient for the same mode order, $Z_{j}$ is the dynamic structural impedance and $A_{Q_{j} Q_{n}}$ is the well-known joint acceptance integral.

The numerical load approximation in Eq. (7) is used for both the full FE model and the WFEbased. The mesh sizes are so that the aliasing frequency is $2000 \mathrm{~Hz}$ and the critical frequency is $580 \mathrm{~Hz}$. The response is computed in any point of the mesh grid. Fig 14 reports the comparison of the results for a specific grid point. The present methodology, through the use of a variable mesh, feasible due to the wave-based method coupled with the transfer matrix in Eq. (8), calculated step-by-step in the frequency domain, extends the accuracy even over the aliasing frequency. On the contrary, as expected, the FEM results become more and more inaccurate above the aliasing frequency. A difference in the modal behaviour between the analytic and numerical model is present. This is addressable to the analytic model being developed to take into account the only bending of the plate, while the FE-based methods, are modelled to take into account a more complete behaviour. Obviously, the possibility to easily refine the solution mesh moves the aliasing frequency to higher one. This explains the results enhancements versus the full FEM ones.

Moreover the present method is much faster than the standard one, thus giving a double advantage for the analysis of these specific problems (Table 3). It is important to underline that, if a variable mesh is used, the elapsed time per frequency is given by an average on the whole frequency band, because the real time is very low when the computed frequency step is far from the upper limit of the frequency band and increases as the frequency increases up to the maximum frequency band. The flexibility of the method allows huge advantages in the easier link among the structural and fluid mesh. It must be emphasized also that all the degrees of freedom, which are 


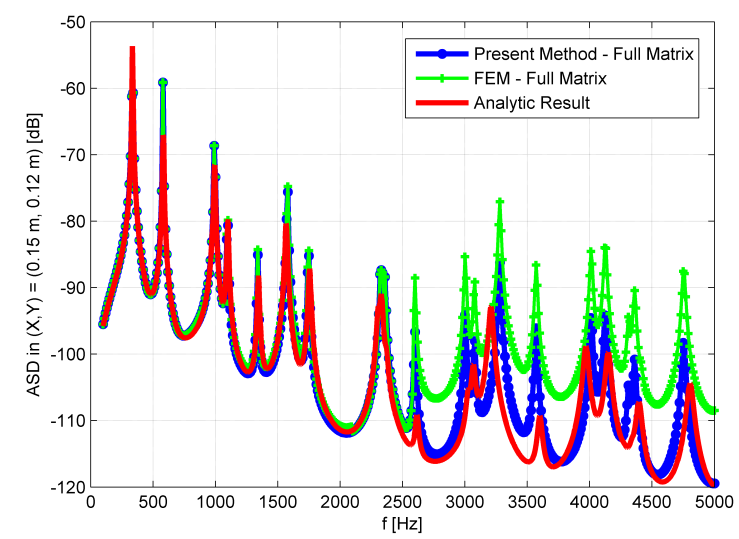

Figure 14: Analytic-Numerical results comparison for a flat panel subjected to TBL - Auto Spectral Density in one point, [dB ref. $1 \mathrm{~m} / \mathrm{s}]$.

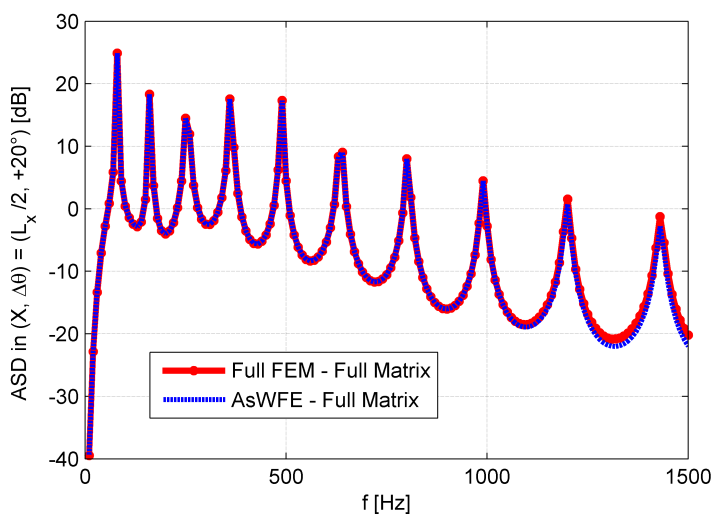

Figure 15: Numerical results comparison for a cylinder subjected to TBL - Auto Spectral Density in one point, [dB ref. $1 \mathrm{~m} / \mathrm{s}$.

not wetted, do not increase the problem size, but, at the same time, the response can be calculated even in non-wetted points.

Damping does not affect the mesh sizes, thus the computational cost, but, on the other hand, it can be modelled, in the framework of the dynamic stiffness matrix approach, through frequencydepending matrices [17, 20, 26. The method, however, has a drawback in the eigenvalue problem, which must be solved at each frequency in order to obtain the wavemodes of the periodic structure. This might be a problem with very complex industrial structures, if an order reduction is not used.

\subsection{Stochastic Response of Axial-symmetric Structures: Cylinder Subjected to TBL}

The cylinder, described in Table 1 is used as a test-case with the Corcos load model, considering the cross-wise distances as the circumference arcs, in the form proposed in [27. The AsWFE method is used and compared with the full FE model.

In this case, the aliasing frequency is $1250 \mathrm{~Hz}$ and, as shown in Fig 15 , the discrepancy among the FE scheme and the present method increases above this frequency. This can be addressed to the loss of validity of Eq. (7), which is circumvented with the present method through the use of variable 
Table 4: Computational cost for a cylindrical structure to TBL load

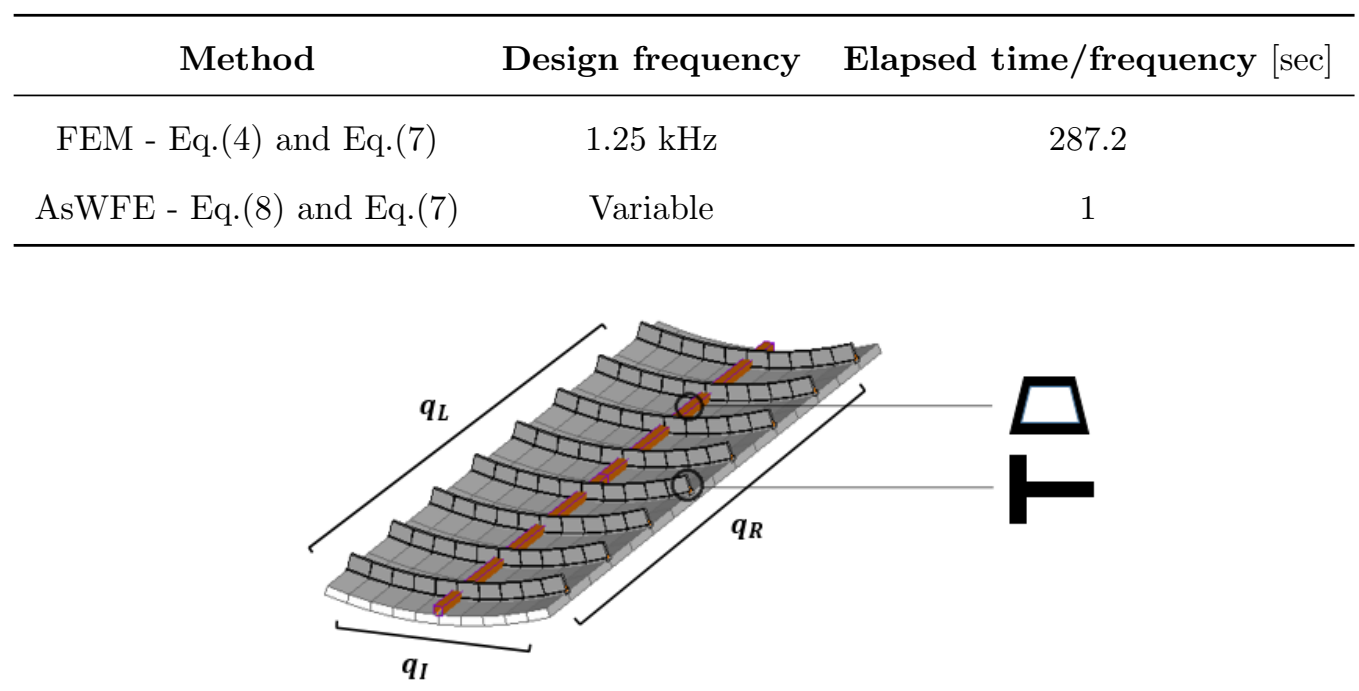

Figure 16: The elementary substructure used to analyse the structural response using the AsWFE method

meshes. The results show a good agreement in the whole frequency range, as expected, because, as previously stated, the validation of the method of calculation of the Green functions guarantees the results for the stochastic cases, assuming that the load description in discrete coordinates is correct.

It is worth underlining also the ease of use of this technique when dealing with pressure loads since the local system of coordinates, which becomes the only reference (lagrangian) when the curvature of the system is simulated, gives the chance of having always one axis normal to the surface so the load translation into nodes is easier that the one needed for classic FE methods and requires a lower size of the load matrix itself.

\subsection{Stochastic Response: Stiffened Cylinder Subjected to TBL}

Singly and doubly stiffened cylinders are often used in many engineering areas, so a stiffened structure is here considered for completeness. The structure is a build up of the cylinder analysed in the previous case and a pattern of frames and stringers. The considered substructure is shown in Fig. 16 and a condensation of the inner dynamics is performed before applying the AsWFE method. A Corcos TBL load model is used to simulate the excitation of the outer surface and the response is calculated for all the nodes of the full FE model with the present technique and the reference method (FEM). The stringers and frames geometrical properties are described in Table 5.

In Fig. 17 the agreement among the curves proves again the accuracy of the method, as Table 6 proves the computational efficiency.

\subsection{Stochastic Response: Complex Axial-symmetric Structure Subjected to DAF}

In this section, the complex axial-symmetric shape (SYLDA model) in Fig. 9 is used as a test-case. However, due to gradient effects, it is not completely correct to apply a Corcos model: 
Table 5: Stiffeners geometrical parameters

\begin{tabular}{ccc}
\hline Data & Circumferential & Longitudinal \\
\hline Height $[\mathrm{mm}]$ & 10.0 & 4.0 \\
Bottom Width [mm] & 5.0 & 3.0 \\
Top Width [mm] & 2.0 & 2.5 \\
Thickness [mm] & 2.0 & 1.0 \\
\hline
\end{tabular}

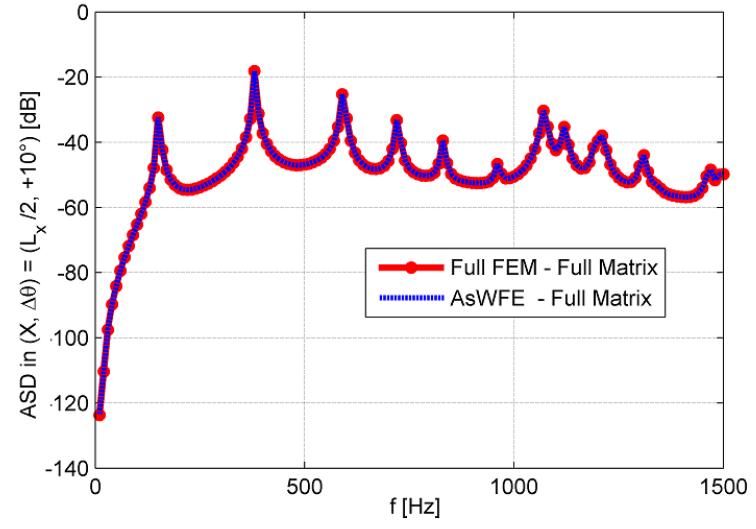

a)

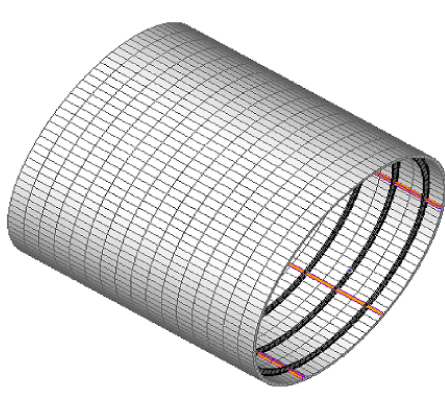

b)

Figure 17: Numerical results comparison for a stiffened cylinder subjected to TBL - Auto Spectral Density in one point (a) and the structure analyzed (b), [dB ref. $1 \mathrm{~m} / \mathrm{s}]$.

Table 6: Computational cost for a stiffened cylindrical structure to TBL load

\begin{tabular}{ccc}
\hline Method & Design frequency & Elapsed time/frequency [sec] \\
\hline FEM - Eq.44 and Eq.47 & $1.5 \mathrm{kHz}$ & 197.8 \\
AsWFE - Eq.8 and Eq. 87 & $1.5 \mathrm{kHz}$ & 1 \\
\hline
\end{tabular}




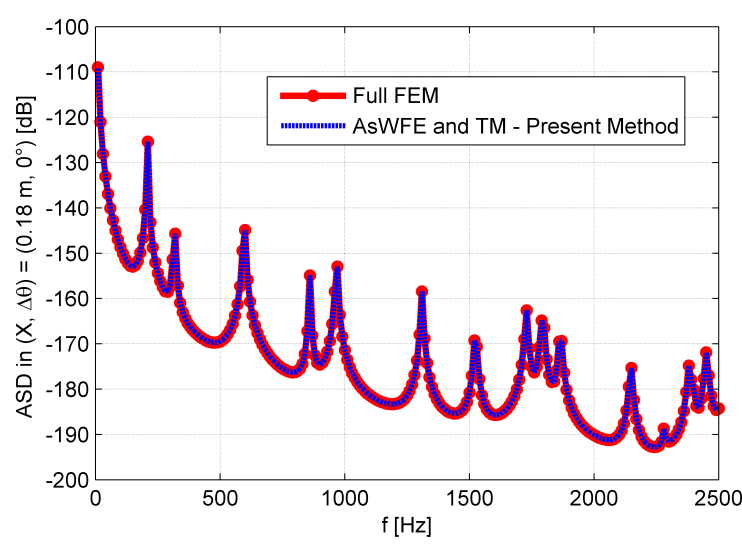

Figure 18: Numerical results for the SYLDA models subjected to DAF load - Auto Spectral Density in one point, [dB ref. $1 \mathrm{~m} / \mathrm{s}$ ].

Table 7: Computational cost for a complex axial-symmetric structure to DAF load

\begin{tabular}{ccc}
\hline Method & Design frequency & Elapsed time/frequency [sec] \\
\hline FEM - Eq.44 and Eq. 47 & $2.5 \mathrm{kHz}$ & 244.8 \\
AsWFE - Eq. 8 and Eq.47 & $2.5 \mathrm{kHz}$ & 1 \\
\hline
\end{tabular}

an incident diffuse field (DAF) is thus simulated. In Fig. 9, the relative substructure used within the present approach is also shown. The DAF is often used to describe the TBL load in the low frequencies (i.e. load description on a space launcher fairing during take-off and climb phases), overestimating the effects for increasing excitation frequency.

Fig. 18 shows the comparison between a full stochastic FE method and the proposed AsWFE with the TM approach. No variable meshes are used in this case: as expected, the two models are equivalent in terms of accuracy. Nevertheless the huge difference of the computational cost is in Table 7. The use of the transfer matrix, in fact, strongly increases the flexibility in terms of selection of the degrees of freedom, both for the target and the wetted, while the wave-based method to evaluate the Green functions allows the use of a reduced model for all the calculation steps.

\section{Conclusions}

This work proposes a numerical methodology for the analysis of the flow-induced vibrations of periodic and axial-symmetric structures. The approach involves a transfer matrix to couple the translated load to the required (target) degrees of freedom. The method through which the Green functions are calculated is a wave finite element method, reformulated in the case of axialsymmetric structures to cope with complex and tapered shapes. A dynamic or static condensation is also possible for complex cross-sectional geometries.

While the analytic methods are limited to very simple cases and the standard FEM approaches can only cope with the low frequency range, the present method allows an extension of the FE- 
based numerical analysis of the flow-induced structural vibrations to higher frequencies within the medium frequency range, for a given computational cost, or a reduced calculation time, given a frequency band of interest. The method uses FE matrices extracted from any commercial code, thus has also the advantage of being able to use all the available FE formulation.

Various test-cases are analysed in order to validate the methodology proposed. The transfer matrix building (Green functions calculation) is validated for the axial-symmetric wave-based formulation, then, the stochastic response of cylinders and complex structures is calculated. The method proves being accurate and much faster than classic FEM, by two orders of magnitude. Depending on the complexity of the structure, the frequency range of analysis and the number of degrees of freedom of the single substructure, the computational cost save can be even higher. The robustness of the technique is proved both with uncorrelated and spatially-correlated loads. Moreover, the possibility to consider the wave propagation through an analytic system of coordinates and, consequently, through the use of variable meshes, gives huge advantages in extending the accuracy to higher frequency bands (over the aliasing frequency) having, at the same time, a great save in cpu time.

The flexibility of the proposed method to link the structural and fluid meshes gives the possibility of using a single substructure to analyse different test-cases. For a given substructure, many parameters can be arbitrary set to specific values: the curvature, the mesh spacing and extension of the structure along the periodicity direction. Within this framework, for example, an entire class of cylinders can be tested by using a single FE model.

The presence of complex structural parts which are not excited does not increase the size of the WFE problem even if the stochastic response can be easily evaluated in such points. Differently, the standard FEM approaches would experience a strong increase in the number of degrees of freedom of the problem.

\section{Acknowledgements}

This project has received funding from the European Unions Horizon 2020 research and innovation program under the Marie Sklodowska-Curie grant agreement No. 675441. The author would like to gratefully acknowledge everyone involved in the VIPER project.

\section{References}

[1] G. Corcos, Resolution of pressure in turbulence, Journal of the Acoustical Society of America 35 (1963) 192-199. doi:/10.1121/1.1918431.

[2] B. Efimtsov, Characteristics of the field of turbulent wall pressure fluctuations at large reynolds numbers, Soviet PhysicsAcoustics 28 (4) (1982) 289-292.

[3] J. F. Williams, Boundary layer pressures and the corcos model: a development to incorporate low wavenumber constraints, Journal of Fluid Mechanics 125 (1982) 9-25. doi:/10.1017/ S0022112082003218. 
[4] A. Smol'yakov, V. Tkachenko, Model of pseudosonic turbulent wall pressures and experimental data, Soviet PhysicsAcoustic 37 (6) (1991) 627-631. doi:/10.1121/1.4960516.

[5] D. Chase, Modelling the wavevector-frequency spectrum of turbulent boundary layer wall pressure, Journal of Sound and Vibration 70 (1) (1953) 29-67. doi:/10.1016/0022-460X (80) 90553-2.

[6] I. Elishakoff, Probabilistic method in theory of structures, John Wiley and Sons (1983) New Yorkdoi:/10.2514/3.48790.

[7] M. Ichchou, B. Hiverniau, B. Troclet, Equivalent rain on the roof loads for random spatially correlated excitations in the mid frequency range, Journal of Sound and Vibration 322 (2009) 926-940. doi:/10.1016/j.jsv.2008.11.050

[8] E. Ciappi, F. Magionesi, S. D. Rosa, F. Franco, Analysis of the scaling laws for the turbulence driven panel responses, Journal of Fluids and Structures 32 (2012) 90-103. doi:/10.1016/ j.jfluidstructs.2011.11.003.

[9] S. D. Rosa, F.Franco, Exact and numerical responses of a plate under a turbulent boundary layer excitation, Journal of Fluids and Structures 24 (2008) 212-230. doi:/10.1016/j. jfluidstructs.2007.07.007.

[10] F. Franco, S. D. Rosa, E. Ciappi, Numerical approximations on the predictive responses of plates under stochastic and convective loads, Journal of Fluids and Structures 42 (2013) 296312. doi:/10.1016/j.jfluidstructs.2013.06.006.

[11] S. D. Rosa, F.Franco, E.Ciappi, A simplified method for the analysis of the stochastic response in discrete coordinates, Journal of Sound and Vibration 339 (2015) 359-375. doi:/10.1016/ j.jsv.2014.11.010.

[12] S. D. Rosa, F.Franco, A scaling procedure for the response of an isolated system with high modal overlap factor, Mechanical Systems and Signal Processing 22 (2008) 1549-1565. doi: $/ 10.1016 /$ j.ymssp.2008.01.007.

[13] L. Brillouin, Wave propagation in periodic structures: Electric filters and crystal lattices, 2nd edition Dover Publications (Mineola, New York). doi:10.1016/S0031-8914(53)80099-6.

[14] D. Mead, Wave propagation in continuous periodic structures: research contributions from southampton, Journal of Sound and Vibration 190 (3) (1996) 495-524. doi:/10.1006/jsvi. 1996.0076 .

[15] E. Manconi, B. R. Mace, Modelling wave propagation in two dimensional structures using finite element analysis, Journal of Sound and Vibration 318(45) (2008) 884-902. doi:/10. $1016 / j \cdot j$ sv.2008.04.039. 
[16] Y. Waki, B. Mace, M. Brennan, Numerical issues concerning the wave and finite element method for free and forced vibrations of waveguides, Journal of Sound and Vibration 327 (2009) 92-108. doi:/10.1016/j.jsv.2009.06.005

[17] C. Droz, C. Zhou, M. Ichchou, J.-P. Laine, A hybrid wave-mode formulation for the vibroacoustic analysis of 2d periodic structures, Journal of Sound and Vibration 363 (2016) 285-302. doi:/10.1016/j.jsv.2015.11.003

[18] C. Droz, J.-P. Laine, M. Ichchou, G. Inquiete, A reduced formulation for the free-wave propagation analysis in composite structures, Composite Structures 113 (2014) 134-144. doi:/10.1016/j.compstruct.2014.03.017.

[19] D. Chronopoulos, M. Ichchou, B. Troclet, O. Bareille, Computing the broadband vibroacoustic response of arbitrarily thick layered panels by a wave finite element approach, Applied Acoustics 77 (2014) 89-98. doi:/10.1016/j.apacoust.2013.10.002

[20] J.-M. Mencik, M. Ichchou, Wave finite elements in guided elastodynamics with internal fluid, International Journal of Solids and Structures 44(7.8) (2007) 2148-2167. doi:/10.1016/j. ijsolstr.2006.06.048

[21] J.-M. Mencik, On the low- and mid-frequency forced response of elastic structures using wave finite elements with one-dimensional propagation, Computers and Structures 88 (2010) 674689. doi:/10.1016/j.compstruc.2010.02.006

[22] V. D'Alessandro, Investigation and assessment of the wave and finite element method for structural waveguides, PhD thesis University of Naples Federico II.

[23] D. Chronopoulos, Prediction of the vibroacoustic response of aerospace composite structures in a broadband frequency range, $\mathrm{PhD}$ thesis Ecole Centrale de Lyon.

[24] J. Renno, B. Mace, Calculating the forced response of cylinders using the wave and finite element method, Journal of Sound and Vibration 333 (2014) 5340-5355. doi:/10.1016/j. jsv.2014.04.042

[25] J. Morsbol, S. Sorokin, Elastic wave propagation in curved flexible pipes, International Journal of Solids and Structures 75-76 (2015) 143-155. doi:/10.1016/j.ijsolstr.2015.08.009.

[26] D. Chronopoulos, B. Troclet, O. Bareille, M. Ichchou, Modeling the response of composite panels by a dynamic stiffness approach, Composite Structures 96 (2013) 111-120. doi:/10. $1016 / j$.compstruct.2012.08.047

[27] Y. Li, Y. Zhang, D. Kennedy, Random vibration analysis of axially compressed cylindrical shells under turbulent boundary layer in a symplectic system, Journal of Sound and Vibrations 406 (2017) 161-180. doi:/10.1016/j.jsv.2017.06.018 